\title{
Effect of Microalgae on Physico-Chemical Properties of Different Dilutions of Untreated and Treated Dairy Industrial Effluent
}

\author{
S.P. Sahana* and Geeta G. Shirnalli \\ Department of Agricultural Microbiology, College of Agriculture, Dharwad, University of \\ Agricultural Sciences, Dharwad, Karnataka, India \\ *Corresponding author
}

\section{A B S T R A C T}

\section{Keywords \\ Dairy, Effluent, \\ Microalgae, Chemical \\ oxygen demand, \\ Chlorella MA-6 \\ Article Info \\ Accepted: \\ 23 March 2018 \\ Available Online: \\ 10 April 2018}

Microalgae have been applied to cultivate in many kinds of wastewater to improve water quality for years. Especially grow microalgae in agro-industrial wastewater, which rich in nitrogen and phosphorus pollutants meanwhile microalgae can be used to reduce the inorganic and organic load of these wastewaters at a minimal cost. The present investigation was aimed in determination of effect of growth of microalgae on physicochemical properties of different dilutions of untreated and treated dairy industrial effluent such as $\mathrm{pH}$, Total solids, Chemical oxygen demand, Nitrate, Phosphate. The results showed that in 40 per cent of untreated effluent and in 100 per cent of treated effluent highest amount of physico-chemical parameters were decreased. Out of five strains used such as Chlorella MA-6, Chlorella MA-14, Botryococcus MA-5, Botryococcus sp., Scenedesmus sp., strain Chorella MA-6 reduced all the parameters significantly compared to all other strains.

\section{Introduction}

Dairy is one of the industries producing wastewater rich in organic matter and thus leading to creation of odorous and high COD containing water. Discharge of dairy plant effluents to the water resources can lead to destruction of aquatic life and other marine creatures, which can provide more food for microbial consortia and causes further oxygen depletion. The $\mathrm{pH}$ of the effluent is alkaline and the organic content is considerably high. The effluent affects the aesthetic value of the receiving water its alkaline $\mathrm{pH}$ causes damage to aquatic life. In dairy wastewaters, nitrogen originates mainly from milk proteins, and is either present in organic nitrogen form such as proteins, urea and nucleic acids, or as ions such as $\mathrm{NH}^{+4}, \mathrm{NO}^{-2}$, and $\mathrm{NO}^{-3}$. Phosphorus is found mainly in inorganic forms such as orthoactive phosphorus $\left(\mathrm{PO}_{3}{ }^{-4}\right)$ and polyactive phosphorus $\left(\mathrm{P}_{2} \mathrm{O}_{4}{ }^{-7}\right)$ as well as in organic forms also. Significant amount of $\mathrm{Na}, \mathrm{Cl}, \mathrm{K}$, $\mathrm{Ca}, \mathrm{Mg}, \mathrm{Fe}, \mathrm{CO}, \mathrm{Ni}$ and $\mathrm{Mn}$ are also always present in dairy wastewater. The presence of high concentration of $\mathrm{Na}$ and $\mathrm{Cl}$ is due to the use of large amount of alkaline cleaners in dairy plant (Harush et al., 2011)

Biological treatment involves microbial degradation and oxidation of waste in the presence of oxygen. Conventional treatment of 
dairy wastewater by aerobic processes includes processes such as activated sludge, trickling filters, and aerated lagoons. The dairy industry uses aerobic or anaerobic treatment, or a combination of both, to treat the wastewater. Aerobic systems require an energy source to provide the oxygen required to assimilate the organic matter. Anaerobic systems have been developed for their ability to treat high strength wastes and the utilization of the methane gas (Bharati and Shinkar, 2013)

Microalgae have been applied to cultivate in many kinds of wastewater to improve water quality for years. Especially grow microalgae in agro-industrial wastewater, which rich in nitrogen and phosphorus pollutants meanwhile microalgae can be used to reduce the inorganic and organic load of these wastewaters at a minimal cost. Using algae has been shown to be a more cost effective way to remove chemical oxygen demand, phosphorus and nitrogen than activated sludge against the traditional waste water treatment processes at ETPs (effluent treatment plants) which involves high energy costs of mechanical aeration to provide oxygen to aerobic bacteria to consume the organic compounds in the waste water.

Bioremediation uses naturally occurring microorganisms (microalgae) to treat wastewater of its nutrients. This method provides an economical and environmentally sustainable and effective treatment method.

Algae are an important bioremediation agent, and are already being used by many wastewater facilities. Algae growth in wastewater treatment ponds contributes to treatment mainly through dissolved oxygen production and nutrient assimilation (Ashish et al., 2012). In the present study the effect of microalgae on physico-chemical properties of dairy effluent was studied.

\section{Materials and Methods}

Untreated and treated dairy wastewater was collected in pre-sterilized bottles from Karnataka Milk Federation unit at Dharwad (Plate 1). Untreated and treated dairy effluents were collected and both were diluted with tap water as 20 per cent $\left(D_{1}-20 \mathrm{ml}\right.$ effluent+ 80 $\mathrm{ml}$ tap water), 40 per cent $\left(\mathrm{D}_{2}-40 \mathrm{ml}\right.$ effluent $+60 \mathrm{ml}$ tap water $), 60$ per cent $\left(\mathrm{D}_{3}-60 \mathrm{ml}\right.$ effluent $+40 \mathrm{ml}$ tap water $), 80$ per cent $\left(\mathrm{D}_{4}\right.$ $80 \mathrm{ml}$ effluent $+20 \mathrm{ml}$ tap water), 100 per cent ( $\mathrm{D}_{5}$ - No dilution)

The diluted water sets viz., $\mathrm{D}_{1}, \mathrm{D}_{2}, \mathrm{D}_{3}, \mathrm{D}_{4}, \mathrm{D}_{5}$ were subjected for analysis of initial physicochemical parameters according to the standard procedures, (APHA, 2005).

\section{Determination of physico-chemical parameters of untreated and treated dairy effluent}

\section{pH}

$\mathrm{pH}$ was estimated by electrometric method. Fifty $\mathrm{ml}$ of each sample was taken separately and the initial $\mathrm{pH}$ was determined by using digital pH meter (Digital pH meter 335).

\section{Chemical Oxygen Demand (COD)}

The COD was estimated by reflux method. Twenty $\mathrm{ml}$ of diluted effluent sample was taken in $250 \mathrm{ml} \mathrm{COD} \mathrm{flask.} \mathrm{Ten} \mathrm{ml}$ of $0.25 \mathrm{~N}$ potassium dichromate (12.26 $\mathrm{g}$ dissolved in $1000 \mathrm{ml}$ distilled water) and $30 \mathrm{ml}$ of sulphuric acid were added and refluxed for two hour on a hot plate. After cooling, $150 \mathrm{ml}$ of distilled water was added. Twenty $\mathrm{ml}$ of refluxed sample was taken in a conical flask and two to three drops of ferroin indicator was added and titrated against $0.25 \mathrm{~N}$ Ferrous ammonium sulphate $(98 \mathrm{~g}$ of $\mathrm{Fe}\left(\mathrm{NH}_{4}\right)_{2}\left(\mathrm{SO}_{4}\right)_{2} \cdot 6 \mathrm{H}_{2} \mathrm{O}$ dissolved in distilled water, $20 \mathrm{ml}$ of concentrated $\mathrm{H}_{2} \mathrm{SO}_{4}$ was 
added and diluted to 1 l) solution. Blank was run by taking twenty $\mathrm{ml}$ of distilled water and using the same chemicals. The COD was calculated using the following formula.

\section{Calculation}

$\operatorname{COD}(\mathrm{mg} / \mathrm{l})=\frac{(\mathrm{a}-\mathrm{b}) \times \mathrm{N} \times 8000}{\mathrm{ml} \mathrm{of} \mathrm{sample}}$

Where,

$\mathrm{a}=\mathrm{ml}$ FAS used for blank

$\mathrm{b}=\mathrm{ml}$ FAS used for sample

$\mathrm{N}=$ Normality of FAS

$8000=$ milli equivalent of $\mathrm{O}_{2} \times 1000$

\section{Total solids}

Total solids were estimated by estimating the amount of total suspended solids and total dissolved solids

\section{Total suspended solids}

Total suspended solids were estimated by gravimetric method. Fifty $\mathrm{ml}$ of a well-mixed sample was taken and filtered through a preweighed filter paper $\left(\mathrm{W}_{1}\right)$.

After filtration the filter paper was dried at $103 \pm 2{ }^{\circ} \mathrm{C}$ until constant weight was obtained. The samples were cooled in desiccators and weighed $\left(\mathrm{W}_{2}\right)$.

\section{Calculation}

Total suspended

$$
\left(\mathrm{W}_{2}-\mathrm{W}_{1}\right)
$$

Solids $(\mathrm{mg} / \mathrm{l})(\mathrm{A})=\frac{}{\mathrm{ml} \text { of sample }} \times 1000$

Where,

$\mathrm{W}_{1}$ : Initial weight; $\mathrm{W}_{2}$ : Final weight

\section{Total dissolved solids}

Total dissolved solids were estimated by gravimetric method. Sample of fifty $\mathrm{ml}$ was transferred to a pre weighed evaporating dish $\left(\mathrm{W}_{1}\right)$. It was evaporated to dryness on steam bath maintained at $80{ }^{\circ} \mathrm{C}$. Evaporated sample was dried for at least one hour in an oven at $180 \pm 2{ }^{\circ} \mathrm{C}$. The samples were cooled in a desiccator and weighed $\left(\mathrm{W}_{2}\right)$.

\section{Calculation}

Total dissolved

$$
\left(\mathrm{W}_{2}-\mathrm{W}_{1}\right)
$$

$$
\text { Solids }(\mathrm{mg} / \mathrm{l})(\mathrm{B})=\frac{}{\mathrm{ml} \text { of sample }} \times 1000
$$

Where, $\mathrm{W}_{1}$ : Initial weight; $\mathrm{W}_{2}$ : Final weight

Total solids calculated by using the formula

Total solids $(\mathrm{mg} / \mathrm{l})=\mathrm{A}+\mathrm{B}$

\section{Nitrate nitrogen}

Nitrate nitrogen content of effluent was estimated by chromotrophic acid (CTA) method. The CTA stock solution of 0.1 per cent was prepared by dissolving $1.84 \mathrm{~g}$ of practical grade CTA disodium salt in $1000 \mathrm{ml}$ of reagent grade sulphuric acid (Specific Gravity-1.84). CTA working solution of 0.01 per cent was prepared by pipetting out $100 \mathrm{ml}$ of 0.1 stock solution, to which $10 \mathrm{ml}$ of concentrated $\mathrm{HCl}$ was added and the volume was made up to $1000 \mathrm{ml}$ with concentrated sulphuric acid. The standard nitrate solution (100 ppm) was prepared using potassium nitrate $(0.7218 \mathrm{~g}$ in $1000 \mathrm{ml})$. A series of working standards were prepared in the range of 2 to $10 \mathrm{ppm}$ and to this $7 \mathrm{ml}$ of CTA working solution was added. The final volume was made up to $25 \mathrm{ml}$. The intensity of yellow colour was measured at $430 \mathrm{~nm}$ using UV visible spectrophotometer (GX-UVS-0227G) 
after 30 minutes of incubation. For the effluents, $3 \mathrm{ml}$ of aliquot was taken in a volumetric flask and to that $5 \mathrm{ml}$ of CTA solution was added and the volume was made up to $25 \mathrm{ml}$.

\section{Calculation} Nitrate

Optical Density at $430 \mathrm{~nm} \times$ aliquot taken Nitrogen $(\mathrm{mg} / \mathrm{l})=\frac{}{\text { Volume made up }(\mathrm{ml})} \times 1000$

\section{Phosphate}

The phosphate of effluent was measured by Vanadomolybdo phosphoric acid method. Vanadomolybdate reagent was prepared by dissolving $25 \mathrm{~g}$ of ammonium molybdate and $1.25 \mathrm{~g}$ of ammonium metavanadate in $600 \mathrm{ml}$ of distilled water. Then $330 \mathrm{ml}$ of concentrated hydrochloric acid was added and the volume was made up to $1000 \mathrm{ml}$. Standard phosphate solution was prepared using potassium dihydrogen phosphate $(0.219 \mathrm{~g}$ in $1000 \mathrm{ml}$ ). A series of standards were prepared to obtain required per cent concentration in the range of 2 to $10 \mathrm{ppm}$. To $35 \mathrm{ml}$ of sample, ten $\mathrm{ml}$ of vanadomolybdate reagent was added and the volume was made up to $50 \mathrm{ml}$. The intensity of the colour was recorded using UV visible spectrophotometer (GX-UVS-0227G) at $490 \mathrm{~nm}$. The phosphorus content in effluent was determined by using following formula.

$$
\mathrm{PO}_{4}(\mathrm{mg} / \mathrm{l})=\frac{\mathrm{mg} \mathrm{P}}{\mathrm{ml} \text { of sample }} \times 1000
$$

Screening of microalgal strains for their growth efficiency in untreated and treated dairy industrial effluent

Strains such as Chlorella MA-6 ( $\left.\mathrm{S}_{1}\right)$, Chlorella MA-14 ( $\left.\mathrm{S}_{2}\right)$, Botryococcus MA-5 $\left(\mathrm{S}_{3}\right)$, Botryococcus sp. $\left(\mathrm{S}_{4}\right)$, Scenedesmus sp.
$\left(\mathrm{S}_{5}\right)$, were chosen from the collection of microalgal strains in the Department of Agricultural Microbiology, University of Agricultural Sciences, Dharwad (Plate 2). Strains were inoculated to the diluted samples separately and the results were interpreted based on their ability to grow in dairy effluent and their ability to affect the physico-chemical properties of dairy industrial effluent. The change in physico-chemical parameters was estimated after three weeks of inoculation.

\section{Results and Discussion}

\section{pH}

The data on initial $\mathrm{pH}$ of untreated and treated diluted effluent is given in table 1 . The $\mathrm{pH}$ of untreated effluent was 6.08, treated effluent was 8.46 and the $\mathrm{pH}$ of water used for dilution was 7.5. On dilution of untreated water the $\mathrm{pH}$ was increased in 20,40,60 and 80 per cent dilution was 7.00, 6.77, 6.61 and 6.25, respectively. The $\mathrm{pH}$ of treated effluent was decreased on dilution with tap water in 20, 40, 60 and 80 per cent was $8.00,8.15,8.26$ and 8.46 , respectively.

The data on change in $\mathrm{pH}$ due to the growth of microalgae is shown in table 2. Significant differences were observed among the type of effluent, dilution of effluent and microalgal strains. But there were no significant difference observed among interactions. However, $\mathrm{pH}$ in the untreated diluted effluent inoculated with different microalgal strains was in the range from 6.34 to 7.06 and in treated effluent ranging from 7.49 to 8.54 .

\section{Total solids}

Total solids were measured in terms of $\mathrm{mg}$ per liter. The data regarding the initial amount total solids of untreated and treated effluent is presented in table 1. Total solids of diluted untreated dairy effluent were 387.27, 774.3, 
961.6, 1348 and $1535.17 \mathrm{mg}$ per liter in 20, $40,60,80$ and 100 per cent dilution, respectively. Total solids of diluted treated dairy effluent were 108.34, 197.6, 268.83, 276.7 and $396.44 \mathrm{mg}$ per liter in 20, 40, 60, 80 and 100 per cent dilution, respectively. Upon dilution, the amount of total solids present in the effluent was decreased upto 74.77 per cent in untreated effluent and 72.67 per cent in treated effluent.

The data in table 3 shows that the changes in amount of total solids present in untreated and treated dairy industrial effluent. Among the types of effluent, dilution of effluent and microalgal strains and in their interactions significant difference was observed in the change in a number of total solids present in dairy industrial effluents.

Among the interactions of type of effluent, dilution of effluent and microalgal strains, the highest amount of total solids was decreased (108.34 to $5.36 \mathrm{mg} / \mathrm{l}(95.05 \%)$ ) in treated effluent $\left(\mathrm{W}_{2}\right)$ with dilution $\mathrm{D}_{1}(20: 80)$ and strain $\mathrm{S}_{1}$ (Chlorella MA-6) whereas lower amount (1535.17 to $1350.29(12.04 \%)$ ) of total solids decreased in untreated effluent $\left(\mathrm{W}_{1}\right)$ with dilution $\mathrm{D}_{5}$ (100:0) and strain $\mathrm{S}_{5}$ (Scenedesmus sp.).

\section{Chemical Oxygen Demand (COD)}

The data on the initial amount of COD of effluent is presented in table 1 and expressed in $\mathrm{mg}$ per liter. The COD of diluted untreated dairy effluent was 367.43, 581.37, 687.67, 787.50 and $898.58 \mathrm{mg}$ per liter respectively for $20,40,60,80$ and 100 per cent dilution. The COD of treated dairy effluent was 153.8 , $265.8,309.4,382.3$ and $465.8 \mathrm{mg}$ per liter in dilution of 20,40,60, 80 and 100 per cent. Chemical oxygen demand of the diluted samples decreased upto 59.10 per cent in untreated effluent and 66.98 per cent in treated effluent as the rate of dilution increased.
The decrease in the amount of COD of untreated and treated dairy industrial effluent is given in table 4. Significant differences were observed among the type of effluent, dilution of effluent, microalgal strains and their interactions. In the interaction of untreated effluent $\left(\mathrm{W}_{1}\right)$ with dilution $\mathrm{D}_{1}$ (20:80) and strain $\mathrm{S}_{1}$ (Chlorella MA-6) the amount of COD reduced was 91.39 per cent (367.43 to $31.64 \mathrm{mg} / \mathrm{l}$ ) which was on par with treated effluent $\left(\mathrm{W}_{2}\right)$ with dilution $\mathrm{D}_{1}(20: 80)$ and strain $S_{1}$ (153.80 to $16.76 \mathrm{mg} / \mathrm{l}$ i.e., $89.10 \%)$, dilution $\mathrm{D}_{2}(40: 60)$ of untreated effluent $\left(\mathrm{W}_{1}\right)$ with strain $\mathrm{S}_{1}$ (581.37 to 65.70 $\mathrm{mg} / \mathrm{l}$ i.e., $88.70 \%)$ and in treated effluent $\left(\mathrm{W}_{2}\right)$ of effluent concentration $\mathrm{D}_{2}$ (40:60) with strain $\mathrm{S}_{1}(265.80$ to $32.33 \mathrm{mg} / \mathrm{l}$ i.e., $87.87 \%)$. Reduction of COD was minimum (898.58 to $374.41 \mathrm{mg} / \mathrm{l}$ i.e., $58.33 \%$ ) in the interaction treatment with untreated effluent $\left(\mathrm{W}_{1}\right)$, effluent concentration $\mathrm{D}_{5}(100: 0)$ and strain $\mathrm{S}_{5}$ (Scenedesmus sp.).

\section{Nitrate content}

The data on initial nitrate content of effluent is shown in table 1 . Nitrate content in untreated diluted dairy wastewater was 7.3, 11.06, $16.14,21.7$ and $34.5 \mathrm{mg}$ per liter respectively in $20,40,60,80$ and 100 per cent dilutions. Nitrate content was 4.16, 7.68, 11.04, 15.19, and $19.28 \mathrm{mg}$ per liter in 20, 40, 60, 80 and 100 per cent of treated dairy effluent, respectively.

As the rate of dilution increased the nitrate content in the effluent also decreased upto 78.84 per cent in untreated effluent and 78.42 per cent in treated effluent.

Change in amount of nitrate present in untreated and treated dairy industrial effluent due to the growth of algae is shown in table 5. A significant difference was observed among the type of effluent, dilution of effluent, microalgal strains and their interactions. 
Table.1 Initial properties of untreated and treated dairy wastewater at various dilution ratio

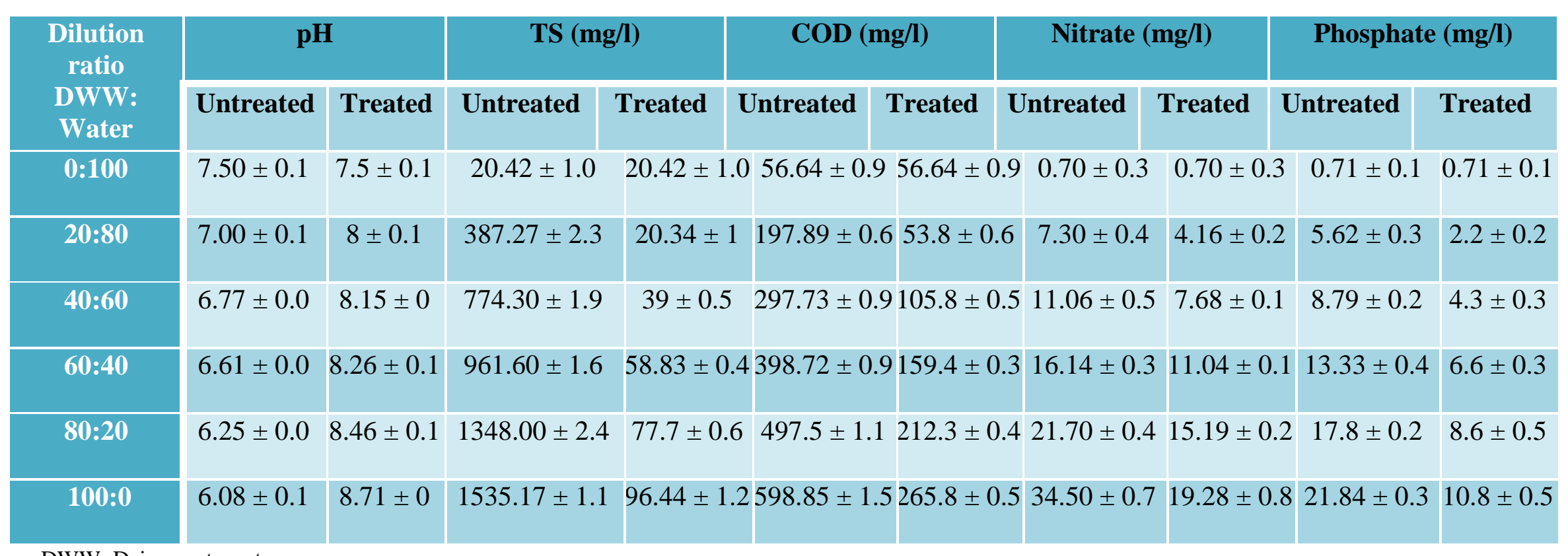

DWW: Dairy waste water 
Table. $2 \mathrm{pH}$ of effluent as influenced by dilution of effluent and inoculation with microalgal strains

\begin{tabular}{|c|c|c|c|c|c|c|c|}
\hline \multicolumn{8}{|c|}{ pH } \\
\hline \multirow{2}{*}{$\begin{array}{l}\text { Type of } \\
\text { effluents }\end{array}$} & \multirow{2}{*}{$\begin{array}{c}\text { Microalgal } \\
\text { strains }\end{array}$} & \multicolumn{6}{|c|}{ Dilution factor } \\
\hline & & $\mathbf{D}_{1}$ & $\mathbf{D}_{2}$ & $\mathbf{D}_{3}$ & $\mathbf{D}_{4}$ & $\mathbf{D}_{5}$ & $\mathbf{W} \times \mathbf{S}$ \\
\hline \multirow{5}{*}{$\mathrm{W}_{1}$} & $\mathbf{S}_{1}$ & 6.63 & 6.82 & 6.93 & 6.99 & 6.34 & 6.74 \\
\hline & $\mathbf{S}_{\mathbf{2}}$ & 6.68 & 6.82 & 6.90 & 7.05 & 6.41 & 6.77 \\
\hline & $\mathbf{S}_{\mathbf{3}}$ & 6.71 & 6.80 & 6.85 & 6.96 & 6.57 & 6.79 \\
\hline & $\mathbf{S}_{4}$ & 6.83 & 6.89 & 6.90 & 7.06 & 6.53 & 6.84 \\
\hline & $\mathbf{S}_{5}$ & 6.76 & 6.83 & 6.89 & 6.97 & 6.61 & 6.81 \\
\hline \multirow[t]{5}{*}{$\overline{W_{2}}$} & $\mathbf{S}_{1}$ & 7.72 & 7.93 & 8.03 & 8.13 & 8.49 & 8.06 \\
\hline & $\mathbf{S}_{2}$ & 7.49 & 7.89 & 7.84 & 8.23 & 8.42 & 7.97 \\
\hline & $\mathbf{S}_{\mathbf{3}}$ & 7.62 & 7.83 & 7.67 & 8.25 & 8.38 & 7.95 \\
\hline & $\mathbf{S}_{4}$ & 7.74 & 7.88 & 7.86 & 8.16 & 8.54 & 8.04 \\
\hline & $\mathbf{S}_{5}$ & 7.81 & 7.83 & 7.96 & 8.25 & 8.42 & 8.06 \\
\hline \multirow[t]{3}{*}{$\mathbf{W} \times \mathbf{D}$} & & & & & & & Mean (W) \\
\hline & $\mathbf{W}_{1}$ & 6.72 & 6.83 & 6.89 & 7.01 & 6.49 & 6.79 \\
\hline & $\mathbf{W}_{2}$ & 7.68 & 7.88 & 7.87 & 8.20 & 8.45 & 8.02 \\
\hline \multirow[t]{6}{*}{$\mathbf{D} \times \mathbf{S}$} & & & & & & & Mean (S) \\
\hline & $\mathbf{S}_{1}$ & 7.18 & 7.38 & 7.48 & 7.56 & 7.42 & 7.40 \\
\hline & $\mathbf{S}_{2}$ & 7.09 & 7.36 & 7.37 & 7.64 & 7.41 & 7.37 \\
\hline & $\mathbf{S}_{\mathbf{3}}$ & 7.17 & 7.32 & 7.26 & 7.60 & 7.46 & 7.37 \\
\hline & $\mathbf{S}_{4}$ & 7.29 & 7.39 & 7.38 & 7.61 & 7.53 & 7.44 \\
\hline & $\mathbf{S}_{5}$ & 7.29 & 7.33 & 7.43 & 7.61 & 7.56 & 7.43 \\
\hline \multicolumn{2}{|l|}{ Mean (D) } & 7.20 & 7.35 & 7.38 & 7.60 & 7.47 & \\
\hline \multicolumn{2}{|c|}{ Factors } & \multicolumn{3}{|c|}{$P(0.01)$} & \multicolumn{3}{|c|}{ S.Em } \\
\hline \multicolumn{2}{|c|}{ Factor (W) } & \multicolumn{3}{|c|}{0.04} & \multicolumn{3}{|c|}{0.01} \\
\hline \multicolumn{2}{|c|}{ Factor (D) } & \multicolumn{3}{|c|}{0.06} & \multicolumn{3}{|c|}{0.02} \\
\hline \multicolumn{2}{|c|}{ Factor $(\mathbf{S})$} & \multicolumn{3}{|c|}{0.06} & \multicolumn{3}{|c|}{0.03} \\
\hline \multicolumn{2}{|c|}{ Interaction $\mathbf{W} \times \mathbf{D}$} & \multicolumn{3}{|c|}{0.09} & \multicolumn{3}{|c|}{0.02} \\
\hline \multicolumn{2}{|c|}{ Interaction $\mathbf{W} \times \mathbf{S}$} & \multicolumn{3}{|c|}{ NS } & \multicolumn{3}{|c|}{0.03} \\
\hline \multicolumn{2}{|c|}{ Interaction $\mathbf{D} \times \mathbf{S}$} & \multicolumn{3}{|c|}{ NS } & & 0.0 & \\
\hline Interactio & $W \times D \times S$ & & NS & & & $0.0^{\circ}$ & \\
\hline $\begin{array}{l}\text { W: Type of ef } \\
\mathrm{W}_{1}: \text { Untreated } \\
\mathrm{W}_{2} \text { : Treated ef }\end{array}$ & $\begin{array}{l}\text { uents } \\
\text { ffluent } \\
\text { uent }\end{array}$ & $\begin{array}{l}\text { D: Diluti } \\
\mathrm{D}_{1}: 20: 80 \\
\mathrm{D}_{2}: 40: 60 \\
\mathrm{D}_{3}: 60: 40 \\
\mathrm{D}_{4}: 80: 20 \\
\mathrm{D}_{5}: 100: 0\end{array}$ & ratio & $\begin{array}{l}\text { S: Mi } \\
\mathrm{S}_{1}: C h \\
\mathrm{~S}_{2}: C h \\
\mathrm{~S}_{3}: B o \\
\mathrm{~S}_{4}: B o \\
\mathrm{~S}_{5}: S c\end{array}$ & $\begin{array}{l}\text { sal stra } \\
\text { a MA- } \\
\text { a MA-1 } \\
\text { ccus } \mathrm{N} \\
\text { ccus } \mathrm{s} \\
\text { smus } \mathrm{sp}\end{array}$ & & \\
\hline
\end{tabular}


Table.3 Total solids of effluent as influenced by dilution of effluent and inoculation with microalgal strains

\begin{tabular}{|c|c|c|c|c|c|c|c|}
\hline \multicolumn{8}{|c|}{ Total solids (mg/) } \\
\hline \multirow{2}{*}{$\begin{array}{l}\text { Type of } \\
\text { effluents }\end{array}$} & \multirow{2}{*}{$\begin{array}{l}\text { Microalgal } \\
\text { strains }\end{array}$} & \multicolumn{6}{|c|}{ Dilution factor } \\
\hline & & $\mathbf{D}_{1}$ & $\mathbf{D}_{2}$ & $\mathbf{D}_{3}$ & $\mathbf{D}_{4}$ & $D_{5}$ & $\mathbf{W} \times \mathbf{S}$ \\
\hline \multirow{5}{*}{$W_{1}$} & $\mathbf{S}_{1}$ & 112.49 & 231.85 & 340.80 & 465.94 & 579.78 & 346.17 \\
\hline & $\mathbf{S}_{2}$ & 193.07 & 387.04 & 579.00 & 769.73 & 966.80 & 579.13 \\
\hline & $\mathbf{S}_{\mathbf{3}}$ & 195.91 & 283.21 & 407.10 & 494.69 & 616.70 & 399.52 \\
\hline & $\mathbf{S}_{4}$ & 224.53 & 312.22 & 462.95 & 508.50 & 687.39 & 439.12 \\
\hline & $S_{5}$ & 267.54 & 538.54 & 808.25 & $1,088.95$ & $1,350.29$ & 810.72 \\
\hline \multirow[t]{5}{*}{$\overline{W_{2}}$} & $\mathbf{S}_{1}$ & 5.36 & 11.37 & 17.32 & 23.13 & 28.96 & 8.06 \\
\hline & $\mathbf{S}_{\mathbf{2}}$ & 9.27 & 19.19 & 29.33 & 38.03 & 48.59 & 7.97 \\
\hline & $\mathbf{S}_{\mathbf{3}}$ & 7.65 & 15.43 & 23.25 & 30.76 & 38.73 & 7.95 \\
\hline & $\mathbf{S}_{4}$ & 10.19 & 21.31 & 32.53 & 42.82 & 52.72 & 8.04 \\
\hline & $\mathbf{S}_{5}$ & 13.53 & 26.62 & 40.97 & 54.38 & 67.91 & 8.06 \\
\hline \multirow[t]{3}{*}{$\mathbf{W} \times \mathbf{D}$} & & & & & & & Mean $(W)$ \\
\hline & $\mathrm{W}_{1}$ & 198.71 & 350.57 & 519.62 & 665.57 & 840.19 & 514.93 \\
\hline & $\mathbf{W}_{2}$ & 9.20 & 18.79 & 28.68 & 37.82 & 47.38 & 28.37 \\
\hline \multirow[t]{6}{*}{$\mathrm{D} \times \mathrm{S}$} & & & & & & & Mean (S) \\
\hline & $\mathbf{S}_{1}$ & 58.92 & 121.61 & 179.06 & 244.54 & 304.37 & 181.70 \\
\hline & $\mathbf{S}_{\mathbf{2}}$ & 101.17 & 203.12 & 304.17 & 403.88 & 507.70 & 304.01 \\
\hline & $\mathbf{S}_{3}$ & 101.78 & 149.32 & 215.18 & 262.73 & 327.72 & 211.35 \\
\hline & $\mathbf{S}_{\mathbf{4}}$ & 117.36 & 166.76 & 247.74 & 275.66 & 370.05 & 235.52 \\
\hline & $\mathbf{S}_{5}$ & 140.54 & 282.58 & 424.61 & 571.67 & 709.10 & 425.70 \\
\hline Mean (D) & & 103.95 & 184.68 & 274.15 & 351.69 & 443.79 & \\
\hline \multicolumn{2}{|c|}{ Factors } & \multicolumn{3}{|c|}{$\mathrm{P}(\mathbf{0 . 0 1 )}$} & \multicolumn{3}{|c|}{ S.Em } \\
\hline \multicolumn{2}{|c|}{ Factor (W) } & \multicolumn{3}{|c|}{0.98} & \multicolumn{3}{|c|}{0.35} \\
\hline \multicolumn{2}{|c|}{ Factor (D) } & \multicolumn{3}{|c|}{1.55} & \multicolumn{3}{|c|}{0.55} \\
\hline \multicolumn{2}{|c|}{ Factor $(\mathbf{S})$} & \multicolumn{3}{|c|}{2.19} & \multicolumn{3}{|c|}{0.78} \\
\hline \multicolumn{2}{|c|}{ Interaction $\mathbf{W} \times \mathbf{D}$} & \multicolumn{3}{|c|}{1.55} & \multicolumn{3}{|c|}{0.55} \\
\hline \multicolumn{2}{|c|}{ Interaction $\mathbf{W} \times \mathbf{S}$} & \multicolumn{3}{|c|}{2.19} & \multicolumn{3}{|c|}{0.78} \\
\hline \multicolumn{2}{|c|}{ Interaction $\mathbf{D} \times \mathbf{S}$} & \multicolumn{3}{|c|}{3.46} & \multicolumn{3}{|c|}{1.23} \\
\hline Interactio & $n W \times D \times S$ & & 4.89 & & & 1.74 & \\
\hline $\begin{array}{l}\text { V: Type of eff } \\
V_{1}: \text { Untreated } \\
V_{2}: \text { Treated eff }\end{array}$ & $\begin{array}{l}\text { luents } \\
\text { ffluent } \\
\text { luent }\end{array}$ & $\begin{array}{l}\text { D: Dilutic } \\
D_{1}: 20: 80 \\
D_{2}: 40: 60 \\
D_{3}: 60: 40 \\
D_{4}: 80: 20 \\
D_{5}: 100: 0\end{array}$ & natio & $\begin{array}{l}\text { S: Mict } \\
\mathrm{S}_{1}: \text { Chl } \\
\mathrm{S}_{2}: \text { Chl } \\
\mathrm{S}_{3}: \text { Bot } \\
\mathrm{S}_{4}: \text { Bot } \\
\mathrm{S}_{5}: \text { Scer }\end{array}$ & $\begin{array}{l}\text { elgal strains } \\
\text { ella MA-6 } \\
\text { ella } \text { MA-14 } \\
\text { ococcus MA-5 } 5 \text { ococcus } \mathrm{sp} \text {. } \\
\text { desmus } \mathrm{sp} \text {. }\end{array}$ & & \\
\hline
\end{tabular}


Table.4 Chemical oxygen demand of effluent as influenced by dilution of effluent and inoculation with microalgal strains

\begin{tabular}{|c|c|c|c|c|c|c|c|}
\hline \multicolumn{8}{|c|}{ Chemical oxygen demand (mg/l) } \\
\hline \multirow{2}{*}{$\begin{array}{l}\text { Type of } \\
\text { effluents }\end{array}$} & \multirow{2}{*}{$\begin{array}{c}\text { Microalgal } \\
\text { strains }\end{array}$} & \multicolumn{6}{|c|}{ Dilution factor } \\
\hline & & $\mathbf{D}_{1}$ & $\mathbf{D}_{2}$ & $\mathbf{D}_{3}$ & $\mathbf{D}_{4}$ & $\mathbf{D}_{5}$ & $\mathbf{W} \times \mathbf{S}$ \\
\hline \multirow{5}{*}{$\mathbf{W}_{1}$} & $\mathbf{S}_{1}$ & 31.64 & 65.70 & 92.60 & 126.00 & 148.94 & 92.98 \\
\hline & $\mathbf{S}_{2}$ & 52.89 & 106.11 & 159.50 & 213.38 & 268.09 & 160.00 \\
\hline & $\mathbf{S}_{\mathbf{3}}$ & 45.60 & 73.39 & 114.64 & 164.07 & 184.34 & 116.41 \\
\hline & $\mathbf{S}_{4}$ & 67.39 & 132.49 & 186.33 & 215.88 & 223.29 & 165.08 \\
\hline & $\mathbf{S}_{5}$ & 74.80 & 147.51 & 222.97 & 295.09 & 374.41 & 222.96 \\
\hline \multirow[t]{5}{*}{$\overline{\mathbf{W}_{2}}$} & $\mathbf{S}_{1}$ & 16.76 & 32.23 & 47.48 & 63.74 & 78.61 & 47.76 \\
\hline & $\mathbf{S}_{2}$ & 28.93 & 53.20 & 79.16 & 106.70 & 132.81 & 80.16 \\
\hline & $\mathbf{S}_{\mathbf{3}}$ & 21.48 & 42.80 & 54.11 & 75.00 & 106.23 & 59.92 \\
\hline & $\mathbf{S}_{4}$ & 29.99 & 64.83 & 84.34 & 119.05 & 119.66 & 83.57 \\
\hline & $\mathbf{S}_{\mathbf{5}}$ & 37.55 & 74.91 & 112.92 & 148.43 & 186.73 & 112.11 \\
\hline \multirow[t]{3}{*}{$\mathbf{W} \times \mathbf{D}$} & & & & & & & Mean $(W)$ \\
\hline & $\mathbf{W}_{1}$ & 54.46 & 105.04 & 155.21 & 202.88 & 239.81 & 151.48 \\
\hline & $\mathbf{W}_{2}$ & 26.94 & 53.59 & 75.60 & 102.58 & 124.81 & 76.71 \\
\hline \multirow[t]{6}{*}{$\mathbf{D} \times \mathbf{S}$} & & & & & & & Mean (S) \\
\hline & $\mathbf{S}_{1}$ & 24.20 & 48.97 & 70.04 & 94.87 & 113.78 & 70.37 \\
\hline & $\mathbf{S}_{\mathbf{2}}$ & 40.91 & 79.66 & 119.33 & 160.04 & 200.45 & 120.08 \\
\hline & $\mathbf{S}_{3}$ & 33.54 & 58.09 & 84.37 & 119.53 & 145.29 & 88.17 \\
\hline & $\mathbf{S}_{4}$ & 48.69 & 98.66 & 135.34 & 167.46 & 171.47 & 124.32 \\
\hline & $\mathbf{S}_{5}$ & 56.18 & 111.21 & 167.94 & 221.76 & 280.57 & 167.53 \\
\hline \multicolumn{2}{|l|}{ Mean (D) } & 40.70 & 79.32 & 115.41 & 152.73 & 182.31 & \\
\hline \multicolumn{2}{|c|}{ Factors } & \multicolumn{3}{|c|}{$P(0.01)$} & \multicolumn{3}{|c|}{ S.Em } \\
\hline \multicolumn{2}{|c|}{ Factor (W) } & \multicolumn{3}{|c|}{0.74} & \multicolumn{3}{|c|}{0.26} \\
\hline \multicolumn{2}{|c|}{ Factor (D) } & \multicolumn{3}{|c|}{1.17} & \multicolumn{3}{|c|}{0.42} \\
\hline \multicolumn{2}{|c|}{ Factor $(\mathbf{S})$} & \multicolumn{3}{|c|}{1.65} & \multicolumn{3}{|c|}{0.59} \\
\hline \multicolumn{2}{|c|}{ Interaction $\mathbf{W} \times \mathbf{D}$} & \multicolumn{3}{|c|}{1.17} & \multicolumn{3}{|c|}{0.42} \\
\hline \multicolumn{2}{|c|}{ Interaction $\mathbf{W} \times \mathbf{S}$} & \multicolumn{3}{|c|}{1.65} & \multicolumn{3}{|c|}{0.59} \\
\hline \multicolumn{2}{|c|}{ Interaction $\mathbf{D} \times \mathbf{S}$} & \multicolumn{3}{|c|}{2.61} & & 0.93 & \\
\hline Interactio & $W \times D \times S$ & & 3.70 & & & 1.32 & \\
\hline $\begin{array}{l}\text { : Type of effl } \\
{ }_{1}: \text { Untreated ef } \\
I_{2}: \text { Treated effl }\end{array}$ & $\begin{array}{l}\text { ents } \\
\text { luent } \\
\text { ent }\end{array}$ & $\begin{array}{l}\text { D: Dilutio } \\
\mathrm{D}_{1}: 20: 80 \\
\mathrm{D}_{2}: 40: 60 \\
\mathrm{D}_{3}: 60: 40 \\
\mathrm{D}_{4}: 80: 20 \\
\mathrm{D}_{5}: 100: 0\end{array}$ & ratio & $\begin{array}{l}\text { S: Micr } \\
\mathrm{S}_{1}: \text { Chlo } \\
\mathrm{S}_{2}: \text { Chlo } \\
\mathrm{S}_{3}: \text { Botr } \\
\mathrm{S}_{4}: \text { Botr } \\
\mathrm{S}_{5}: \text { Scen }\end{array}$ & $\begin{array}{l}\text { lgal strai } \\
\text { lla } \mathrm{MA}-6 \\
\text { lla } \mathrm{MA}-14 \\
\text { coccus } \mathrm{M} \\
\text { coccus } \mathrm{sp} . \\
\text { esmus } \mathrm{sp} .\end{array}$ & & \\
\hline
\end{tabular}


Table.5 Nitrate content of effluent as influenced by dilution of effluent and inoculation with microalgal strains

\begin{tabular}{|c|c|c|c|c|c|c|c|}
\hline \multicolumn{8}{|c|}{ Nitrate content (mg/l) } \\
\hline \multirow{2}{*}{$\begin{array}{l}\text { Type of } \\
\text { effluents }\end{array}$} & \multirow{2}{*}{$\begin{array}{c}\text { Microalgal } \\
\text { strains }\end{array}$} & \multicolumn{6}{|c|}{ Dilution factor } \\
\hline & & $\mathbf{D}_{1}$ & $\mathbf{D}_{2}$ & $\mathbf{D}_{3}$ & $\mathbf{D}_{4}$ & $\mathbf{D}_{5}$ & $\mathbf{W} \times \mathbf{S}$ \\
\hline \multirow{5}{*}{$\mathbf{W}_{1}$} & $\mathbf{S}_{1}$ & 0.59 & 1.28 & 2.25 & 3.08 & 2.89 & 2.02 \\
\hline & $\mathbf{S}_{2}$ & 1.21 & 2.47 & 3.91 & 5.11 & 6.21 & 3.78 \\
\hline & $\mathbf{S}_{\mathbf{3}}$ & 1.05 & 1.41 & 2.39 & 4.27 & 4.17 & 2.66 \\
\hline & $\mathbf{S}_{4}$ & 2.17 & 2.95 & 3.21 & 4.13 & 7.25 & 3.94 \\
\hline & $\mathbf{S}_{5}$ & 1.37 & 3.48 & 5.32 & 7.36 & 8.59 & 5.23 \\
\hline \multirow[t]{5}{*}{$\mathbf{W}_{2}$} & $\mathbf{S}_{1}$ & 0.00 & 0.43 & 1.53 & 1.88 & 1.89 & 1.15 \\
\hline & $\mathbf{S}_{\mathbf{2}}$ & 0.00 & 1.49 & 2.02 & 2.51 & 3.22 & 1.85 \\
\hline & $\mathbf{S}_{3}$ & 0.00 & 1.09 & 1.91 & 2.11 & 3.20 & 1.66 \\
\hline & $\mathbf{S}_{\mathbf{4}}$ & 0.00 & 1.76 & 1.60 & 2.73 & 4.32 & 2.08 \\
\hline & $\mathbf{S}_{5}$ & 0.00 & 1.93 & 2.94 & 3.63 & 4.91 & 2.68 \\
\hline \multirow[t]{3}{*}{$\mathbf{W} \times \mathbf{D}$} & & & & & & & Mean (W) \\
\hline & $\mathbf{W}_{1}$ & 1.28 & 2.32 & 3.41 & 4.79 & 5.82 & 3.53 \\
\hline & $\mathbf{W}_{2}$ & 0.00 & 1.34 & 2.00 & 2.57 & 3.51 & 1.88 \\
\hline \multirow[t]{6}{*}{$\mathbf{D} \times \mathbf{S}$} & & & & & & & Mean (S) \\
\hline & $\mathbf{S}_{1}$ & 0.29 & 0.86 & 1.89 & 2.48 & 2.39 & 1.58 \\
\hline & $\mathbf{S}_{\mathbf{2}}$ & 0.61 & 1.98 & 2.96 & 3.81 & 4.72 & 2.82 \\
\hline & $\mathbf{S}_{\mathbf{3}}$ & 0.53 & 1.25 & 2.15 & 3.19 & 3.69 & 2.16 \\
\hline & $\mathbf{S}_{4}$ & 1.08 & 2.35 & 2.40 & 3.43 & 5.79 & 3.01 \\
\hline & $\mathbf{S}_{5}$ & 0.69 & 2.70 & 4.13 & 5.49 & 6.75 & 3.95 \\
\hline Mean (D) & & 0.64 & 1.83 & 2.71 & 3.68 & 4.67 & \\
\hline \multicolumn{2}{|c|}{ Factors } & \multicolumn{3}{|c|}{$\mathbf{P}(\mathbf{0 . 0 1})$} & \multicolumn{3}{|c|}{ S.Em } \\
\hline \multicolumn{2}{|c|}{ Factor (W) } & \multicolumn{3}{|c|}{0.09} & \multicolumn{3}{|c|}{0.03} \\
\hline \multicolumn{2}{|c|}{ Factor (D) } & \multicolumn{3}{|c|}{0.14} & \multicolumn{3}{|c|}{0.05} \\
\hline \multicolumn{2}{|c|}{ Factor (S) } & \multicolumn{3}{|c|}{0.20} & \multicolumn{3}{|c|}{0.07} \\
\hline \multicolumn{2}{|c|}{ Interaction $\mathbf{W} \times \mathbf{D}$} & \multicolumn{3}{|c|}{0.14} & \multicolumn{3}{|c|}{0.05} \\
\hline \multicolumn{2}{|c|}{ Interaction $\mathbf{W} \times \mathbf{S}$} & \multicolumn{3}{|c|}{0.20} & \multicolumn{3}{|c|}{0.07} \\
\hline \multicolumn{2}{|c|}{ Interaction $\mathbf{D} \times \mathbf{S}$} & \multicolumn{3}{|c|}{0.32} & \multicolumn{3}{|c|}{0.11} \\
\hline Interactio & $W \times D \times S$ & & 0.45 & & & 0.1 & \\
\hline $\begin{array}{l}\text { W: Type of eff } \\
\mathrm{W}_{1}: \text { Untreated } \\
\mathrm{W}_{2}: \text { Treated eff }\end{array}$ & $\begin{array}{l}\text { uents } \\
\text { ffluent } \\
\text { uent }\end{array}$ & $\begin{array}{l}\text { D: Dilu } \\
\mathrm{D}_{1}: 20: 8 \\
\mathrm{D}_{2}: 40: 6 \\
\mathrm{D}_{3}: 60: 4 \\
\mathrm{D}_{4}: 80: 2 \\
\mathrm{D}_{5}: 100:\end{array}$ & ratio & $\begin{array}{l}\text { S: Mi } \\
\mathrm{S}_{1}: C h \\
\mathrm{~S}_{2}: C h \\
\mathrm{~S}_{3}: B o \\
\mathrm{~S}_{4}: B o \\
\mathrm{~S}_{5}: S c\end{array}$ & $\begin{array}{l}\text { al stra } \\
a \text { MA- } \\
a \text { MA-1 } \\
\text { ccus } \mathrm{N} \\
\text { ccus } \mathrm{s} \\
\text { smus } \mathrm{sp}\end{array}$ & & \\
\hline
\end{tabular}


Table.6 Phosphate content of effluent as influenced by dilution of effluent and inoculation with microalgal strains

\begin{tabular}{|c|c|c|c|c|c|c|c|}
\hline \multicolumn{8}{|c|}{ Phosphate content (mg/l) } \\
\hline \multirow{2}{*}{$\begin{array}{l}\text { Type of } \\
\text { effluents }\end{array}$} & \multirow{2}{*}{$\begin{array}{c}\text { Microalgal } \\
\text { strains }\end{array}$} & \multicolumn{6}{|c|}{ Dilution factor } \\
\hline & & $\mathbf{D}_{1}$ & $\mathbf{D}_{2}$ & $\mathbf{D}_{3}$ & $\mathbf{D}_{4}$ & $\mathbf{D}_{5}$ & $\mathbf{W} \times \mathbf{S}$ \\
\hline \multirow{5}{*}{$\mathbf{W}_{1}$} & $\mathbf{S}_{1}$ & 1.12 & 2.38 & 3.75 & 5.26 & 6.37 & 3.78 \\
\hline & $\mathbf{S}_{\mathbf{2}}$ & 2.36 & 4.34 & 6.33 & 8.94 & 10.11 & 6.42 \\
\hline & $\mathbf{S}_{\mathbf{3}}$ & 1.96 & 3.47 & 4.59 & 6.32 & 7.25 & 4.72 \\
\hline & $\mathbf{S}_{4}$ & 3.04 & 5.45 & 7.29 & 9.96 & 9.50 & 7.05 \\
\hline & $\mathbf{S}_{5}$ & 3.20 & 6.13 & 9.18 & 12.35 & 15.20 & 9.21 \\
\hline \multirow[t]{5}{*}{$\overline{\mathbf{W}_{2}}$} & $\mathbf{S}_{1}$ & 0.00 & 1.33 & 2.32 & 2.72 & 2.89 & 1.85 \\
\hline & $\mathbf{S}_{\mathbf{2}}$ & 1.19 & 2.76 & 3.54 & 5.32 & 6.32 & 3.83 \\
\hline & $\mathbf{S}_{\mathbf{3}}$ & 0.00 & 1.77 & 2.77 & 3.27 & 4.25 & 2.41 \\
\hline & $\mathbf{S}_{4}$ & 1.41 & 2.90 & 5.75 & 5.70 & 4.69 & 4.09 \\
\hline & $\mathbf{S}_{5}$ & 1.78 & 3.11 & 4.90 & 6.29 & 8.17 & 4.85 \\
\hline \multirow[t]{3}{*}{$\mathbf{W} \times \mathbf{D}$} & & & & & & & Mean (W) \\
\hline & $\mathbf{W}_{1}$ & 2.34 & 4.36 & 6.23 & 8.57 & 9.69 & 6.24 \\
\hline & $\mathbf{W}_{2}$ & 0.88 & 2.37 & 3.86 & 4.66 & 5.26 & 3.41 \\
\hline \multirow[t]{6}{*}{$\mathbf{D} \times \mathbf{S}$} & & & & & & & Mean (S) \\
\hline & $\mathbf{S}_{1}$ & 0.56 & 1.86 & 3.04 & 3.99 & 4.63 & 2.81 \\
\hline & $\mathbf{S}_{2}$ & 1.77 & 3.55 & 4.94 & 7.13 & 8.21 & 5.12 \\
\hline & $\mathbf{S}_{\mathbf{3}}$ & 0.98 & 2.62 & 3.68 & 4.80 & 5.75 & 3.57 \\
\hline & $\mathbf{S}_{4}$ & 2.23 & 4.18 & 6.52 & 7.83 & 7.10 & 5.57 \\
\hline & $\mathbf{S}_{5}$ & 2.49 & 4.62 & 7.04 & 9.32 & 11.68 & 7.03 \\
\hline \multicolumn{2}{|l|}{ Mean (D) } & 1.61 & 3.37 & 5.04 & 6.61 & 7.48 & \\
\hline \multicolumn{2}{|c|}{ Factors } & \multicolumn{3}{|c|}{$P(0.01)$} & \multicolumn{3}{|c|}{ S.Em } \\
\hline \multicolumn{2}{|c|}{ Factor (W) } & \multicolumn{3}{|c|}{0.12} & \multicolumn{3}{|c|}{0.04} \\
\hline \multicolumn{2}{|c|}{ Factor (D) } & \multicolumn{3}{|c|}{0.19} & \multicolumn{3}{|c|}{0.07} \\
\hline \multicolumn{2}{|c|}{ Factor $(\mathbf{S})$} & \multicolumn{3}{|c|}{0.27} & \multicolumn{3}{|c|}{0.09} \\
\hline \multicolumn{2}{|c|}{ Interaction $\mathbf{W} \times \mathbf{D}$} & \multicolumn{3}{|c|}{0.19} & \multicolumn{3}{|c|}{0.07} \\
\hline \multicolumn{2}{|c|}{ Interaction $\mathbf{W} \times \mathbf{S}$} & \multicolumn{3}{|c|}{0.27} & \multicolumn{3}{|c|}{0.09} \\
\hline \multicolumn{2}{|c|}{ Interaction $\mathbf{D} \times \mathbf{S}$} & \multicolumn{3}{|c|}{0.42} & & 0.15 & \\
\hline Interactio & $\mathbf{W} \times \mathbf{D} \times \mathbf{S}$ & & 0.59 & & & 0.21 & \\
\hline $\begin{array}{l}\mathbf{N}: \text { Type of efflu } \\
N_{1}: \text { Untreated ef } \\
N_{2}: \text { Treated efflu }\end{array}$ & $\begin{array}{l}\text { nts } \\
\text { uent } \\
\text { nt }\end{array}$ & $\begin{array}{l}\text { D: Dilutio } \\
\mathrm{D}_{1}: 20: 80 \\
\mathrm{D}_{2}: 40: 60 \\
\mathrm{D}_{3}: 60: 40 \\
\mathrm{D}_{4}: 80: 20 \\
\mathrm{D}_{5}: 100: 0\end{array}$ & & $\begin{array}{l}\text { S: Micl } \\
\mathrm{S}_{1}: \text { Chlc } \\
\mathrm{S}_{2}: \text { Chlc } \\
\mathrm{S}_{3}: \text { Botr } \\
\mathrm{S}_{4}: \text { Botr } \\
\mathrm{S}_{5}: \text { Scer }\end{array}$ & $\begin{array}{l}\text { al strai } \\
\text { a MA-6 } \\
\text { a MA-1 } \\
\text { ccus } \mathrm{M} \\
\text { ccus } \mathrm{sp} \\
\text { mus sp. }\end{array}$ & & \\
\hline
\end{tabular}

In the interaction of treated effluent $\left(\mathrm{W}_{2}\right)$ with dilution $\mathrm{D}_{1}(20: 80)$ and all strains decreased the amount of nitrate by 100 per cent which was significantly higher over all the other treatments. Removal of nitrate was minimum (21.70 to $7.36 \mathrm{mg} / \mathrm{l}$ i.e., $66.08 \%$ ) in untreated effluent $\left(\mathrm{W}_{1}\right)$ with dilution $\mathrm{D}_{4}(80: 20)$ and strain $\mathrm{S}_{5}$ (Scenedesmus sp.). 


\section{Phosphate content}

The phosphate content of untreated and treated effluent is presented in table 1 . The amount of phosphate in $20,40,60,80$ and 100 per cent of untreated effluent was 5.62, $8.79,13.33,17.8$ and $21.84 \mathrm{mg}$ per liter, respectively. Phosphate content of diluted treated dairy effluent was $2.2,4.3,6.6,8.6$ and $10.8 \mathrm{mg}$ per liter in 20, 40, 60, 80 and 100 per cent, respectively. The phosphate content of effluent was decreased upto 74.26 per cent in untreated effluent and 79.62 per cent in treated effluent as the rate of dilution increased.

Table 6 represents the data regarding amount of phosphate removed in dairy industrial effluents. In the interaction of treated effluent $\left(\mathrm{W}_{2}\right)$ of effluent concentration $\mathrm{D}_{1}(20: 80)$ and strain $\mathrm{S}_{1}$ (Chlorella MA-6) amount of phosphate reduced by 100 per cent which is significantly higher over all other interactions. The minimum reduction in the amount of phosphate 24.35 per cent (10.80 to $8.17 \mathrm{mg} / \mathrm{l}$ ) was recorded in treated effluent $\left(\mathrm{W}_{2}\right)$ and dilution $\mathrm{D}_{5} \quad(100: 0)$ and the strain $\mathrm{S}_{5}$ (Scenedesmus sp.).

In the present investigation, the performance of microalgal culture in untreated and treated dairy wastewater was evaluated. The dairy industry has adopted activated sludge method for treating the wastewater. The suitability of untreated and treated wastewater for the growth of microalgae was evaluated by analyzing the initial physicochemical parameters.

In the present study initial $\mathrm{pH}$ of untreated dairy effluent was 6.08 while the treated effluent had $8.71 \mathrm{pH}$. Acidic nature of wastewater was due to break down of milk lactose into lactic acid (Medhat and Usama, 2004). Other authors had observed the $\mathrm{pH}$ of untreated dairy effluent in the range of 4.5 to
9 and treated effluent in the range of 7.0 to 8.5 (Noorjahan et al., 2004; Tikariha and Sahu, 2014; Singh et al., 2014).

Total solids in untreated and treated dairy industrial effluents were in the range of 2426 to $890 \mathrm{mg} / \mathrm{l}$ and 318 to $520 \mathrm{mg} / \mathrm{l}$ respectively (Noorjahan et al., 2004; Kolhe and Pawar, 2011; Shivsharan et al., 2013; Singh et al., 2014). Total solids of untreated effluent and treated effluent in this study were $1535.17 \mathrm{mg} / \mathrm{l}$ and $396.44 \mathrm{mg} / \mathrm{l}$ respectively. Total solids were composed mainly of carbonates, bicarbonates, chlorides, sulfate, phosphate, nitrate, $\mathrm{Ca}, \mathrm{Mg}, \mathrm{Na}, \mathrm{K}, \mathrm{Mn}$ and organic matter. Stills and other particles polluting water would increase the concentration of total solids (Kolhe and Pawar, 2011).

The COD value obtained for the untreated and treated effluent was 898.58 and $465.80 \mathrm{mg} / \mathrm{L}$ respectively which might have been due to the presence of inorganic salts. The COD value was much bigger than the BOD value indicating that the wastewater was suitable for the growth of microalgae (Tikariha and Sahu, 2014). Other authors Noorjahan et al., (2004), Shivsharan et al., (2013), Singh et al., (2014) observed the range of COD from 780 to 1210 $\mathrm{mg} / \mathrm{l}$ in untreated effluent and 260 to $490 \mathrm{mg} / \mathrm{l}$ in treated effluent.

Nitrogen is a very important component of the dairy factory wastewaters. Some protein will be lost to the waste streams. Bacteria convert the nitrogen in proteins to the inorganic forms including ammonia, nitrite and nitrate ions. Each of these inorganic forms of nitrogen has different environmental effects (Bharati and Shinkar, 2013). The presence of nitrogen in wastewater during discharge could be an undesirable factor because it has ecological impacts and can affect public health. Nitrate itself is not toxic; its conversion to nitrite is a concern in the domain of public health 
(Sedlak, 1991). The nitrate content of untreated and treated effluent was in the range of 18 to $46 \mathrm{mg} / \mathrm{l}$ and 10 to $34 \mathrm{mg} / \mathrm{l}$, respectively (Longhurst et al., 2000; Noorjahan et al., 2004; Shivsharan et al., 2013; Singh et al., 2014). In the present investigation, nitrate nitrogen content was $34.45 \mathrm{mg} / \mathrm{l}$ and $19.28 \mathrm{mg} / \mathrm{l}$ in untreated and treated wastewater, respectively.

Neha et al., (2013) claimed that phosphate was mainly contributed through detergents and soaps widely used for cleaning purposes in milk processing unit. In the present study, values for phosphate were $21.84 \mathrm{mg} / \mathrm{l}$ and $10.8 \mathrm{mg} / \mathrm{l}$ in untreated and treated water respectively. Noorjahan et al., (2004), Shivsharan et al., (2013), Singh et al., (2014), observed phosphate content in the range of 17 to $34 \mathrm{mg} / \mathrm{l}$ and 8 to $22 \mathrm{mg} / \mathrm{l}$ in untreated and treated effluent, respectively.

Change in physicochemical parameters of untreated and treated effluent due to the growth of microalgae

During phycoremediation process, $\mathrm{pH}$ level of effluent become neutral or around neutrality. The microalgae reduce dissolved $\mathrm{CO}_{2}$ concentrations through photosynthesis which, in turn raises the $\mathrm{pH}$ level. The inorganic nutrients normally used by microalgae are $\mathrm{CO}_{2}$ and bicarbonate (Borowitzka, 1998), the latter requiring the enzyme carbonic anhydrase to convert it to $\mathrm{CO}_{2}$. The microalgal culture treated effluents showed an increase in $\mathrm{pH}$ in the range of 6.34 to 7.06 but there is no significant difference among the treatments.

Total solids of the effluent decreased upon treatment with microalgae, which was due to the utilization of various nutrients by microalgae. There was a reduction in the levels of solids because there could be a conversion of the total suspended solids already present in the effluent into dissolved materials for algal uptake and assimilation (Rao et al., 2011). A maximum of 95.05 per cent decrease in total solids was observed with the strain Chlorella MA-6. Ayodhya et al., (2013) with Chlorella vulgaris and Cho et al., (2011) with Chlorella sp. 227 also observed similar results.

COD was used to measure a number of inorganic compounds in wastewater. The reduction of $\mathrm{COD}$ indicated that microalgae could utilize inorganic carbon in the wastewater as a source of energy and as a substrate for cell growth (Wang et al., 2012). In this study, reduction of COD was 91 per cent by the isolate Chlorella MA-6 which was similar to the results observed by Sreekanth et al., (2014), Choi (2015), Cho et al., (2011) using Chlorella sp. (90 to $96 \%$ reduction).

All forms of nitrogen are taken up as a nutrient by the micro alga for their growth and development, although the most common nitrogen compounds assimilated by microalgae are nitrate $\left(\mathrm{NO}^{3-}\right)$ and ammonium $\left(\mathrm{NH}_{4}{ }^{+}\right)$. In our study, Chlorella MA-6 was able to reduce nitrate nitrogen substantially compared to all other strains. Ayodhya et al., (2013) using Chlorella vulgaris and Cho et al., (2011) using Chlorella sp. 227 also observed similar results.

Phosphate removal by microalgae during phycoremediation is due to the utilization of phosphorus for growth. The phosphorus, which is used in the algal cells mainly for the production of phospholipids, adenosine triphosphates (ATP) and nucleic acids, gets assimilated as inorganic orthophosphate, preferably as $\mathrm{H}_{2} \mathrm{PO}_{4}{ }^{-}$or $\mathrm{HPO}_{4}{ }^{2-}$ (Becker, 1994). The chemical stripping of phosphorus may be regarded as an advantageous sideeffect of the algal growth, with enhanced phosphorus removal as a result. The investigation revealed that the phosphate 
removal efficiency of Chlorella MA-6 was nearly 100 per cent. The removal of phosphate could be considered as assimilation by the microalgae rather than the coagulation and adsorption of inorganic phosphates due to the $\mathrm{pH}$ of broth which was lower than 8 (Zhou et al., 2011).

\section{References}

APHA, 2005, Standard Methods for the Examination of Water and Wastewater; $21^{\text {st }}$ Edition, American Public Health Association/American Water Works Association/Water Environment Federation, Washington DC.

Ashish B, Monica B, Juhi P. (2012). Potential of Treated Dairy Waste Water for the Cultivation of Algae and Waste Water Treatment by Algae. Universal Journal of Environmental Research and Technology. Vol. 2, Issue 1: 101-104

Ayodhya, K., Ibe., S. N. and Abu, G. O., 2013, Economically effective potential of Chlorella spp. for biomass and lipid production. J. Microbiol. Biotechnol. Res., 2(1); 35-45.

Becker, E. W., 1994, Microalgae Biotechnology and Microbiology. Cambridge University Press, Cambridge.

Bharati S. and Shinkar. N (2013). Comparative Study of Various Treatments for Dairy Industry Wastewater. Journal of Engineering (IOSRJEN) Vol. 3, Issue 8 ISSN: 22503021, pp-ISSN: 2278-8719

Borowitzka, M. A., 1998, Limits to growth. In: Wong YS and Tam NFY (eds.) Wastewater Treatment with Algae. Springer Verlag, New York. pp 203226.

Cho, S., Luong, T. T., Lee, D., You-Kwan, O., Lee, T., 2011, Reuse of effluent water from a municipal wastewater treatment plant in microalgae cultivation for biofuel production. Bioresour. Technol., 102:8639-8645.

Choi, H., 2015, Dairy wastewater treatment using microalgae for potential biodiesel application. Environ. Eng. Res., 21(4): 393-400.

Harush D.; Hampannavar U and Mallikarjunaswami M. (2011). Treatment of dairy wastewater using aerobic biodegradation and coagulation. International Journal of Environmental Sciences and Research. College of Engineering and Technology, India. Vol. 1, No. 1, 2011, pp. 23-26.

Kolhe, A.S. and Pawar, V. P., 2011, Physicochemical analysis of effluents from dairy industry. Recent Res. Sci. Technol., 3(5): 29-32.

Longhurst, R., Roberts, A. and O'Connor, M., 2000, Farm dairy effluent: a review of published data on chemical and physical characteristics in New Zealand. N. Z. J. Agric. Res. 43 (1):7-14.

Medhat M. A. S. and Usama F. M., 2004, Anaerobic Digestion technology for industrial waste water treatment, 8th International water Technology Conference, Alexandria, Egypt, 817833.

Neha, S., Chatterjee, S. and Bhatnagar, P., 2013, An evaluation of physicochemical properties to assess quality of treated effluents from Jaipur dairy. Int. J. Chem. Environ. Pharm. Res., 4(3):5458.

Noorjahan, V. S., Sharief, D. and Dawood, N., 2004, Characterization of dairy effluent. J. Ind. Pollut. Control., 20 (1):131 -136.

Rao, P. H., Ranjith, K. R., Raghavan, B. G., Subramanian, V. V. and Sivasubramanian, V., 2011, Application of phycoremediation technology in the treatment of wastewater from a leatherprocessing chemical manufacturing facility. Water., 37 (1):7-14. 
Sedlak, R. 1991, Phosphorus and nitrogen removal from municipal wastewater: Principles and practice (2nd edn.). Lewis Publisher, Ann Arbor, Michigan.

Shivsharan, V. S., Minal, W. and Khetmalas, M. B., 2013, Characterization of dairy effluents by physicochemical parameters. Br. Biotechnol. J., 3(4): 575-580.

Singh, N. B., Singh, R. and Manzer, M. I., 2014, Waste water management in dairy industry: pollution abatement and preventive attitudes. Int. J. Sci. Environ. Technol., 3(2):672 - 683 .

Sreekanth, D., Pooja, K., Seeta, Y., Himabindu, V. and Reddy P. M., 2014, Bioremediation of dairy wastewater using microalgae for the production of biodiesel. Int. J. Sci. Eng. Adv. Technol., 2(11):783-791.

Tikariha, A. and Sahu, O., 2014, study of characteristics and treatments of dairy industry waste water. J. Appl. Environ. Microbiol., 2(1): 16-22.

Wang, H., Xiong, H., Hui, Z. and Zeng, X. 2012, Mixotrophic cultivation of Chlorella pyrenoidosa with diluted primary piggery wastewater to produce lipids. Bioresour. Technol., 104:215220.

Zhou, W., Li, Y., Min, M., Hu, B., Chen, P. and Ruan, R., 2011, Local bioprospecting for high-lipid producing microalgal strains to be grown on concentrated municipal wastewater for biofuel production. Bioresour. Technol. 102(13): 6909-6919.

\section{How to cite this article:}

Sahana, S.P. and Geeta G. Shirnalli. 2018. Effect of Microalgae on Physico-Chemical Properties of Different Dilutions of Untreated and Treated Dairy Industrial Effluent. Int.J.Curr.Microbiol.App.Sci. 7(04): 2979-2993. doi: https://doi.org/10.20546/ijcmas.2018.704.338 